\title{
HOLOMORPHIC APPROXIMATION ON REAL-ANALYTIC SUBMANIFOLDS OF A COMPLEX MANIFOLD
}

\author{
R. O. WELLS, JR.
}

Let $X$ be a complex manifold, and let $K$ be a compact subse $t$ of $X$. Then we will say that $K$ is holomorphic if there is a sequence $U_{i}$ of open Stein submanifolds of $X$ such that $U_{i+1} \subset U_{i}$ and

$$
K=\bigcap_{i=1}^{\infty} L_{i}^{+}
$$

(we will use the terminology of Gunning and Rossi [2]). Let $C(K)$ be the uniform algebra of continuous complex-valued fucntions on $K$ with respect to the maximum norm on $K$. Let $A_{0}(K)$ be the subalgebra of $C(K)$ obtained by requiring that $f \in A_{0}(K)$ if and only if $f$ is the restriction to $K$ of a holomorphic function defined in a neighborhood of $K$. We let $A(K)$ be the completion of $A_{0}(K)$ in $C(K) . A(K)$ is the uniform algebra of holomorphic functions on $K$. Let $S(A(K))$ be the spectrum of $A(K)$. A basic question is: when does $A(K)=C(K)$ (cf. Bishop [1])?

The purpose of this paper is to prove the following

THEOREM. If $M$ is a real-analytic compact submanifold of $X$ with no complex tangent vectors, then $A(M)=C(M)$.

This will follow immediately from the following two lemmas.

Leмma 1. Let $M$ be a compact $C^{\infty}$ submanifold of $X$ with no complex tangent vectors, then $M$ is holomorphic.

REMARK. This theorem and the idea for its proof were suggested to me by L. Hörmander.

Proof. Taking local coordinates $\left(z_{1}, \cdots, z_{n}\right)$ in a neighborhood of $p \in M$, with $p=(0, \cdots, 0)$, we can express $M$ in the following manner near $p$. Let $z_{j}=x_{j}+i y_{j}, j=1, \cdots, n$, and suppose $\operatorname{dim}_{r} M=k$. Set $x=\left(x_{1}, \cdots, x_{k}\right), y=\left(y_{1}, \cdots, y_{k}\right), Z=\left(z_{k+1}, \cdots, z_{n}\right)$. Then we can write $M$ as an embedding of the form

$$
y \rightarrow\left(\begin{array}{l}
g(y)+i y \\
h(y)
\end{array}\right) \in \mathbf{C}^{n},
$$

where

Received by the editors March 10, 1966 and, in revised form, May 1, 1966. 


$$
\begin{aligned}
& g(y)=\left(g_{1}(y), \cdots, g_{k}(y)\right), \\
& h(y)=\left(h_{k+1}(y), \cdots, h_{n}(y)\right)
\end{aligned}
$$

are respectively real and complex-valued (vector-valued) $C^{\infty}$ functions defined in a neighborhood of zero in $R^{k}$, and vanishing to second order there. Set

$$
\phi=|x-g(y)|^{2}+|Z-h(y)|^{2} .
$$

We can calculate the complex Hessian of $\phi$ by noting that

$$
x_{j}=\left(z_{j}+\bar{z}_{j}\right) / 2
$$

and

$$
|h(y)|=O\left(|y|^{2}\right),|g(y)|=O\left(|y|^{2}\right) .
$$

We obtain

$$
\begin{aligned}
\phi_{z_{j} z_{j}} & =\frac{1}{2}+O(|x|+|y|), & & j=1, \cdots, k \\
& =1, & & j=k+1, \cdots, n ; \\
\phi_{z_{i} \bar{z}_{j}} & =0, & & i \neq j, \quad i \text { or } j>k \\
& =O(|y|), & & i \neq j, \quad i, j \leqq k .
\end{aligned}
$$

Hence it follows that for $|x|+|y|$ sufficiently small the eigenvalues of the matrix $\left(\phi_{i_{i} i_{j}}\right)$ will be positive. Thus we can find an $\epsilon>0$ so that $\phi$ is strongly plurisubharmonic in $[|z|<\epsilon]=N$. We also have that $\phi$ vanishes in $N$ only on $M$, and $d \phi=0$ on $N \cap M$.

By compactness of $M$ we can find a finite number of open sets $\left\{N_{j}\right\}$ whose union covers $M$, and such that there is a $C^{\infty}$ function $\phi_{j}$ for each $N_{j}$, satisfying the above properties. If $\left\{\alpha_{j}\right\}$ is a $C^{\infty}$ partition of unity subordinate to $\left\{N_{j}\right\}$, then it follows that $\phi=\sum \alpha_{j} \phi_{j}$ is a $C^{\infty}$ function defined and strongly plurisubharmonic on a neighborhood $U$ of $M$, and which vanishes in $U$ only on $M$. It follows (cf. [5, p. 469]) that there is a sequence $\epsilon_{j} \rightarrow 0$ and that $U_{j}=\left[\phi<\epsilon_{j}\right]$ is a sequence of open Stein submanifolds of $X$ whose intersection is $M$.

Lemma 2. Let $M$ be a real-analytic compact submanifold of a complex manifold $X$. If $M$ is holomorphic and has no complex tangent vectors, then $A(M)=C(M){ }^{1}$

Proof. Since $M$ is holomorphic $M$ is contained in some Stein mani-

1 This type of result was suggested to me by H. Rossi. 
fold $U$, open in $X$. Then $H(U)$, the algebra of holomorphic functions on $U$, separates points in $U$, and hence $A(K)$ separates points on $K$. Thus, since $A(K)$ is closed under uniform limits it will follow from the Stone-Weierstrass theorem that $A(K)=C(K)$ if we know that $f \in A(K)$ implies $\bar{f} \in A(K)$, (i.e., the algebra is self-adjoint).

Therefore it suffices to solve the following problem. Suppose $F$ is holomorphic in a neighborhood of $M$, and let $f=\bar{F} \mid M$, we want to find a function $G$ holomorphic in a neighborhood of $M$ so that $G \mid M=f$. To construct $G$ we have to go through several steps. Let $\operatorname{dim}_{r} M=k$ and $\operatorname{dim}_{c} X=n$.

First we note that since $M$ has no complex tangent vectors, then $k \leqq n$. (If $M$ is orientable, then this would follow from the holomorphicity of $M$, independent of the assumption about complex tangent vectors, cf. Browder [3].)

We now construct a "complexification" of $M$, that is a complex submanifold of $X$ whose "real axis" is $M$.

$M$ is defined in local coordinates near $p \in M$ as a mapping,

$$
\phi: B \subset R^{k} \rightarrow X, \quad \phi(0)=P,
$$

where $\phi$ is real-analytic and $B$ is open in $R^{n} . \phi$ is then the restriction to $B$ of a holomorphic mapping

$$
\tilde{\phi}: \tilde{B} \subset C^{k} \rightarrow X, \quad \tilde{\phi}(0)=\mathrm{p},
$$

where $\widetilde{B}$ is open in $C^{k}$.

Let $R_{1}, \cdots, R_{k}$ be a basis for $T_{p}$, the real tangent space to $M$ at $p$. Let $J$ be the automorphism of $\tau_{p}$, the real tangent space to $X$ at $p$, which gives the complex structure to $\tau_{p}$ induced by the complex structure on $X$. Then the set of real vectors $A=\left\{R_{1}, \cdots, R_{k}, J R_{1}, \cdots, J R_{k}\right\}$ is a set of $2 k$ linearly independent tangent vectors to $X$ at $p$. This follows from the assumption that $M$ has no complex tangent vectors.

We then have a holomorphic map $\tilde{\phi}$ which is nondegenerate at 0 , since $A$ is a set of $2 k$ linearly independent tangent vectors to $\tilde{\phi}(\tilde{B})$ at $p$. Then $\tilde{\phi}(\widetilde{B})$ defines in a neighborhood $N$ of $p$ a complex submanifold of $X$ which contains $M \cap N$ as a submanifold. This we can do for each such set of local coordinates on $M$. We obtain then a $k$-dimensional complex submanifold $V$ of $X$ which contains $M$ as a real submanifold. $V$ is defined in $U$, where $U$ is some neighborhood of $M$. By the holomorphicity of $M$, we may assume that $U$ is an open Stein submanifold of $X$.

We now return to $f$, given above. Let $N$ be a neighborhood of $p \in M$ in which we have local coordinates, $\left(t_{1}, \cdots, t_{k}\right)$. Since $f$ is realanalytic in $\left(t_{1}, \cdots, t_{k}\right)$ we have that $f$ is the restriction to $B \subset R^{k}$ 
of a holomorphic function $F_{N}\left(\zeta_{1}, \cdots, \zeta_{k}\right)$, defined in $\widetilde{B} \subset C^{k}$ where $\left(\zeta_{1}, \cdots, \zeta_{k}\right)$ are local coordinates for $V \cap N$. Thus we can extend $f$ holomorphically from a neighborhood of $p$ in $M$ to a neighborhood of $p$ in $V$, by the real-analyticity of $f$ and $M$. On the intersection of two such neighborhoods the extensions to $V$ must agree since they agree on $M$. Hence, we can choose a smaller neighborhood $U$ of $M$ (which we still require to be Stein) in which $V$ is defined, so that we can extend $f$ from $M$ to a holomorphic function on $V$. But by the extension theorem for Stein manifolds, (see [2]) all holomorphic functions defined in $V$ may be extended to $U$. Thus there exists a holomorphic function $G$, defined in $U$, with $G \mid M=f$.

Q.E.D.

Corollary. Let $\Gamma$ be a compact real-analytic 1-manifold on a complex manifold $X$, then $A(\Gamma)=C(\Gamma)$, and hence $S(A(\Gamma))=\Gamma$, where $S(A(\Gamma))$ is the spectrum of $A(\Gamma)$.

Proof. A 1-manifold on $X$ has no complex tangent vectors. The second conclusion follows from the fact that $S(C(\Gamma))=\Gamma$.

REMARK 1. If $\Gamma$ is the real-analytic diffeomorphic image of $S^{1}$, the unit circle, in $\boldsymbol{C}^{n}$, then the corollary above contrasts with the results of Wermer [4] on the spectrum of the uniform algebra of polynomials on $\Gamma, P(\Gamma)$. In that case $S(P(\Gamma))=\Gamma$ if and only if $\Gamma$ was not the boundary of a Riemann surface in $C^{n}$ (with perhaps multiple points).

REMARK 2. It should be possible to remove the hypothesis of realanalyticity, but the method of proof would have to be entirely different. Hörmander has suggested a way of doing this by methods arising from partial differential equations.

\section{BIBLIOGRAPHY}

1. E. Bishop, Uniform algebras, Proceeding of the Conference on Complex Analysis, Minneapolis, 1964, pp. 272-281, Springer-Verlag, New York, 1965.

2. R. C. Gunning and H. Rossi, Analytic functions of several complex variables, Prentice-Hall, New York, 1965.

3. A. Browder, Cohomology of maximal ideal spaces, Bull. Amer. Math. Soc. 67 (1961), 515-516.

4. J. Wermer, The hull of a curve in $C^{n}$, Ann. of Math. 68 (1958), 550-561.

5. H. Grauert, On Levi's problem and the imbedding of real-analytic manifolds, Ann. of Math. 68 (1958), 460-472.

RICE UNIVERSITY 